\title{
Reproducibility of cerebral artery Doppler measurements
}

\author{
B Moorthy, P R Colditz, K N Ives, D G Rees, W G van't Hoff, P L Hope
}

\begin{abstract}
Intraobserver and interobserver variability were calculated for the measurement of cerebral artery pulsatility indices by five observers who made two recordings on each of 12 stable preterm infants. Variations in pulsatility indices of up to $0 \cdot 21$ were found; no measurement was below 0.55 .
\end{abstract}

There have been many studies of the cerebral circulation of newborn infants with Doppler techniques since the report by Bada $e t$ al in 1979 , in which they used the Pourcelot or pulsatility index. ${ }^{1}$ Pulsatility index is defined as (S-D)/S, where $S$ is systolic and $D$ is diastolic flow velocity. ${ }^{1}$ It has been suggested that a low pulsatility index $(<0.55)$ during the first five days of life is a useful prognostic sign, with a high sensitivity for the prediction of death or disability, in infants who have been subjected to severe birth asphyxia. ${ }^{2}$ The aim of this study was to assess the reproducibility of measurement of pulsatility indices by clinical staff using a commercial duplex scanner.

Patients and methods

Twelve stable preterm infants were each studied within a period of one hour by five observers, using a duplex Doppler scanner (Ultramark 4, Advanced Technical Laboratories). A real time image was obtained in the parasagittal plane, and a Doppler signal obtained at $5 \mathrm{MHz}$ from the anterior cerebral artery anterior to the corpus callosum. Each observer made two separate recordings from the anterior cerebral artery of each infant, and stored the flow velocity waveforms on a hard copy. The hard copies were kept by one of the authors who, after collection of the data was complete, issued each observer with the 24 recordings he had made from the 12 infants. Observers were therefore unaware which were paired results from the same infant. Systolic and diastolic velocities were measured by ruler on the hard copy. Measurements were made to the nearest millimetre and the pulsatility index was calculated as the mean (S-D)/S of at least three waveforms.

Analysis of variance was used to study the 60 first observations by each observer on each infant. ${ }^{3}$ Estimates of the SD of both the intraobserver and interobserver variability were calculated, making the reasonable assumption of no interaction between baby and observer.
Median and range of values for pulsatility indices of 12 infants

\begin{tabular}{|c|c|c|}
\hline \multirow[t]{2}{*}{ Case No } & \multicolumn{2}{|c|}{ Pulsatility indices } \\
\hline & Median & $\begin{array}{l}\text { Range of } \\
\text { five values }\end{array}$ \\
\hline $\begin{array}{r}1 \\
2 \\
3 \\
4 \\
5 \\
6 \\
7 \\
8 \\
9 \\
10 \\
11 \\
12\end{array}$ & $\begin{array}{l}0.81 \\
0.82 \\
0.71 \\
0.76 \\
0.75 \\
0.78 \\
0.74 \\
0.73 \\
0.79 \\
0.81 \\
0.75 \\
0.78\end{array}$ & $\begin{array}{l}0.77-0.85 \\
0.72-0.93 \\
0.63-0.78 \\
0.73-0.83 \\
0.70-0.83 \\
0.75-0.85 \\
0.69-0.80 \\
0.60-0.80 \\
0.75-0.81 \\
0.75-0.88 \\
0.69-0.76 \\
0.73-0.81\end{array}$ \\
\hline
\end{tabular}

\section{Results}

The table shows the medians and ranges of values obtained by the five observers from each of the 12 infants. There was considerable variation between observers, the most extreme being between 0.72 and 0.93 . None of the observers recorded a pulsatility index below the 0.55 threshold suggested by Archer et al. ${ }^{2}$ The estimates of the SDs associated with the intraobserver and interobserver variances were 0.046 and 0.030 , respectively.

These values may be interpreted as follows: (1) Of the differences between two independent measurements made by the same observer on the same patient, $95 \%$ will be less than 0.13 $(=2.83 \times 0.046)$ or $17 \%$ when expressed as a percentage of the mean pulsatility index. ${ }^{4}$ (2) Of the differences between two independent measurements made by two observers on the same patient, $95 \%$ will be less than 0.16 $\left(=2.83 \times \sqrt{\left.0.046^{2}+0.030^{2}\right)}\right.$, or $20 \%$ as a percentage of the mean pulsatility index.

Analysis of the differences between the duplicate determinations by each observer showed that these differences were less than would be predicted if each was subjected independently to the calculated degree of intraobserver variability. The two measurements were therefore not truly independent, and the duplicate measurements were not used in the analysis.

\section{Discussion}

Previous studies of the reproducibility of measurement of pulsatility indices have been limited to comparison of results obtained by two or three experienced observers. ${ }^{2} 56$ As cerebral artery Doppler studies are now useful clinically 
as well as in research, we were interested to find out how reproducible the technique was when used in routine clinical practice. We therefore used a commercially available duplex scanner, and measurements were made by the five people who were responsible for the routine ultrasound surveillance of high risk newborn infants on our nursery. Two of these observers were research personnel and three were in clinical service posts; three had less than one year's experience in ultrasound and Doppler techniques.

If the mean pulsatility index for all observers is taken as the most accurate estimate of the 'true' pulsatility index, then this small sample did not contain any infant with a pulsatility index below 0.55 . It was therefore not possible to determine the risk of a true low pulsatility index being missed by an individual observer. There was no false positive measurement of a pulsatility index less than 0.55 . Incomplete insonnation of a vessel can give the false impression of low or absent diastolic flow and therefore a high pulsatility index, whereas the finding of high diastolic flow and low pulsatility index is much less likely to be the result of observer error. There is therefore less chance that a clinically important error would be made in neonatal cerebral artery Doppler studies than in obstetric scanning, where absent or reduced diastolic flow is the main diagnostic feature.
This study showed that interobserver variability did not exceed intraobserver variability in the measurement of pulsatility index using a duplex Doppler scanner. In some infants, however, measurements of pulsatility index varied widely, and it would therefore seem sensible not to make clinical judgments on a single pulsatility index estimate. This small study was confined to preterm infants, none of whom had a pulsatility index in the pathological range. Reproducibility of measurement of pulsatility indices over a wider range of normal and abnormal values should be established if the pulsatility index is to be used as a prognostic indicator.

1 Bada HS, Hajiar W, Chua C, Sumner DS. Non-invasive diagnosis of neonatal asphyxia and intraventricular haemorrhage by Doppler ultrasound. 7 Pediatr 1979;95:775-9.

2 Archer LNJ, Levene MI, Evans DH Cerebral artery Doppler ultrasonography for prediction of outcome after perinatal asphyxia. Lancet 1986;ii:1116-7.

3 Huitson A. The analysis of variance: a basic course. London: Charles Griffin 1980 .

4 Bland M. An introduction to medical statistics. Oxford: Oxford University Press, 1987

5 Winberg P, Dahlstrom A, Lundell B. Reproducibility of intracranial Doppler flow velocimetry. Acta Paediatr Scand intracranial Doppler flow

6 Hancock PJ, Goldberg RN, Bancalari E, Hill MC, Castillo T Limitation of pulsatility index as a diagnostic tool in the newborn. Crit Care Med 1983;11:186-8.

\title{
Virilisation of female preterm infants
}

\author{
P C Midgley, D Azzopardi, N Oates, J C L Shaw, J W Honour
}

\begin{abstract}
Two cases of hypertrophy of the clitoris in premature girls are reported; this was associated with persistently high concentrations of adrenal fetal zone androgens.
\end{abstract}

Isolated hypertrophy of the clitoris in neonates is known to be associated with congenital adrenal hyperplasia, administration of androgens to the mother in the second and third trimesters of pregnancy, ${ }^{1}$ and maternal androgen secreting tumours. We report two cases of progressive hypertrophy of the clitoris developing after birth, and associated with persistent excretion of high concentrations of $3 \beta-\mathrm{OH}-5$-ene steroids, produced by the fetal adrenal zone and which are known to be weak androgens. This association has not previously been reported.

\section{Case reports}

CASE 1

A girl, birth weight $640 \mathrm{~g}$, was one of twins delivered at 24 weeks' gestation for antepartum haemorrhage; the mother was aged 24, para

London WCIE 6AU.

Accepted 31 January 1990
The surviving twin developed hyaline membrane disease, and was ventilated for five weeks, remaining oxygen dependent for a further seven weeks. Apnoea of prematurity was treated with theophylline from 4 to 10 weeks. She had a patent ductus arteriosus, which failed to close with indomethacin, and led to treatment with digoxin and frusemide from 9 weeks until spontaneous closure at 11 weeks. She had recurrent hyponatraemia and required sodium supplements.

Clitoromegaly was noted at 4 weeks of age (fig 1A). She had a left inguinal hernia with a palpable gonad (a macroscopically normal ovary was later found at herniotomy). Ultrasound showed a normal uterus and adrenals. The karyotype was normal female. Plasma $17 \alpha-\mathrm{OH}$ progesterone was $27 \cdot 2 \mathrm{nmol} / 1$ at 7 weeks, $61 \cdot 8$ $\mathrm{nmol} / \mathrm{l}$ at 9 weeks, and $11 \cdot 2 \mathrm{nmol} / \mathrm{l}$ at 20 weeks. Adrenocorticotrophic hormone (ACTH) was $4.8 \mathrm{pmol} / \mathrm{l}$ at 11 weeks and $6.6 \mathrm{pmol} / 1$ at 20 weeks (immunoradiometric assay, Eurodiagnostics, Netherlands). The plasma cortisol on five occasions, when not receiving hydrocortisone, ranged from $<69 \mathrm{nmol} / \mathrm{l}$ to $316 \mathrm{nmol} / \mathrm{l}$ and after corticotrophin stimulation increased to $>1380$ nmol/l at 60 minutes. Plasma dehydroepian- 